\title{
Roles of dopamine receptors and their antagonist thioridazine in hepatoma metastasis
}

This article was published in the following Dove Press journal:

OncoTargets and Therapy

22 June 2015

Number of times this article has been viewed

\author{
Meiling Lu',* \\ Jinghua $\mathrm{Li}^{1}, *$ \\ Zaili Luo ${ }^{2,3, *}$ \\ Shuai Zhang ${ }^{3}$ \\ Shaobo Xue' \\ Kesheng Wang' \\ Yan Shi ${ }^{4}$ \\ Cunzhen Zhang ${ }^{3}$ \\ Haiyang Chen ${ }^{3}$ \\ Zhong $\mathrm{Li}^{1,5}$
}

'Central Laboratory, The 10th People's Hospital, Tongji University, Shanghai, People's Republic of China; ${ }^{2}$ International Cooperation Laboratory on Signal Transduction, Eastern Hepatobiliary Surgery Institute/Hospital, The Second Military Medical University, Shanghai, People's Republic of China; ' Institution of Biomedical Sciences, Fudan University, Shanghai, People's Republic of China;

${ }^{4}$ Department of Gastroenterology, The I0th People's Hospital, Tongji University, Shanghai, People's Republic of China; ' ${ }^{5}$ Zhangjiang Center for Translational Medicine, Shanghai, People's Republic of China

*These authors contributed equally to this work

\begin{abstract}
Tumor metastasis is the most common cause of death and poor prognosis for cancer patients. Therapeutics that prevent tumor metastasis are the key to prolonging the lifespan of cancer patients. Cancer stem cells are believed to be critical in the metastatic process. Recently, drug screening for cancer stem cells reports that antipsychotic drugs displayed potential anticancer activity. Thioridazine, one of the antipsychotic drugs for dopamine receptors (DRs), is shown to induce the differentiation of cancer stem cells in leukemic disease and breast cancer, but it is not known if this drug would affect liver cancer. In this study, expression of DR5 was higher in tumors than in nontumor adjacent tissues, while DR1 was lower in human hepatocellular carcinoma (HCC) than those in the adjacent tissues. Other DRs were very low or undetectable. Treatment of HCC cells with thioridazine displays a dose-dependent response in HCC cell lines SNU449, LM3, and Huh7. Thioridazine treatment reduced cell viability and sphere formation of $\mathrm{HCC}$ cell lines through induction of G0/G1 cell cycle arrest and suppression of stemness genes CD133, OCT4, and EpCam. It also inhibited cell migration via suppression of epithelial-mesenchymal transition (EMT)-related genes such as twist2 and E-cadherin. Thioridazine-pretreated LM3 cells decreased the capacity of tumorigenesis in nude mice. Taken together, our data suggest that thioridazine may have the potential role in treatment of HCC.
\end{abstract}

Keywords: antipsychotic, thioridazine, HCC, dopamine receptors, metastasis

\section{Introduction}

Hepatocellular carcinoma (HCC) is the sixth most common malignant cancer in the world, and the third leading cause of cancer related deaths worldwide. ${ }^{1}$ Current therapies for diagnostic HCC include surgical resection, liver transplantation, chemotherapy, transarterial chemoembolization (TACE), and radiofrequency ablation. ${ }^{2}$ But unresectable HCC seems more chemoresistant to most currently available chemotherapeutic agents, ${ }^{3}$ and metastasis is the predominant cause of death in the most of cancer patients. So far, we still lack a novel therapeutic target for effective inhibition of liver metastasis.

Thioridazine (10-[2-(1-methyl-2-piperidyl)ethyl]-2-methylthiophenothiazine, THIO), a potent antipsychotic and antianxiety agent is a phenothiazine derivative. It is extensively used to treat psychotic disorders such as psychosis and schizophrenia. In the case of advanced tumor, the drug is widely used for the treatment of tumor-linked sweating and depression. ${ }^{4,5}$ Studies have revealed that antipsychotics have antiproliferative activity and promote apoptosis in gastric cancer, cervical cancer, endometrial cancer, breast cancer, neuroblastoma, glioma, leukemia, and ovarian cancer. ${ }^{6-11}$ Further research disclosed that THIO induced cell cycle (G0/G1) arrest and served as an inhibitor of PI3K/AKT signaling to downregulate the cell cycle regulator Cyclin D1 and CDK4 and upregulate p21, p16, and p-CDC25A in ovarian cancer. ${ }^{11}$ Studies
Correspondence: Zhong $\mathrm{Li}$ Central Laboratory, The 10th People's Hospital, Tongji University, 30I Middle Yanchang Road, Shanghai 200072,

People's Republic of China

Tel +86 2l 36050164

Email lizhongsh@yahoo.com 
on cervical and endometrial cancer cells also points to the involvement of PI3K/AKT/mTOR pathway in THIO induced apoptosis, ${ }^{7}$ whereas THIO-induced gastric cancer apoptosis is in a caspase-dependent manner. ${ }^{6}$ THIO is also found to be a novel antiangiogenic protein that acts through suppressing the alphavbeta3/FAK/mTOR signaling pathway. ${ }^{12}$ Recently, screening of drugs for inhibition of tumors revealed that anitpsychotics showed potential inhibition of cancer and provided a promising direction of treatment of tumors. THIO targets breast and colon cancers and achieves a synergistic effect with other antiproliferative drugs such as doxorubicin (DOX) and sertraline. ${ }^{13,14}$ Reports on tumor reversion have shown that sertraline and THIO target translationally controlled tumor protein (TPT1/TCTP), a key player in reprogramming malignant cells, and inhibit its binding to MDM2, restoring wild-type p53 function. ${ }^{15}$ Recently, THIO was found to selectively target leukemic cancer stem cells but not normal blood stem cells. ${ }^{16}$ Cancer stem cells are believed to be critical in tumor metastasis. Therefore, it is necessary to explore whether application of THIO would be a new approach for inhibition of tumor metastasis.

In the present study, by using several HCC cell lines in cell assays and animal models, we investigated the expression levels of dopamine receptors (DRs) and dopamine in HCC tissues and the effects of THIO on these cell lines and xenograft tumor in nude mice. The results demonstrate that THIO inhibits proliferation, migration, and tumorigenesis of HCC.

\section{Materials and methods Cell lines}

LM3 cells were transferred from cell bank of Zhongshan Hospital, Fudan University Medical College with the material transfer agreement in 2012. Huh7 and SNU449 cell lines were purchased from American Type Culture Collection. Human liver cell line L02 was obtained from Cell Bank of Chinese Academy of Science, People's Republic of China. All of cells were routinely cultured in (Dulbecco's Modified Eagle's Medium) DMEM (Hyclone, Logan, UT, USA) supplemented with 10\% fetal bovine serum (FBS) (Life Technologies, Carlsbad, CA, USA) in a humidified incubator containing $5 \% \mathrm{CO}_{2}$, at $37^{\circ} \mathrm{C}$. THIO was purchased from Sigma Chemical Co. (Sigma-Aldrich, St Louis, MO, USA) and dissolved in DMSO (dimethyl sulfoxide).

\section{MTT assay for cell viability}

Cells were incubated in a 96-well plate at a density of $4 \times 10^{3}$ per well, with or without THIO for 3 days. Concentrations of THIO were 5,10 , or $15 \mu \mathrm{M}$, respectively. Each point was analyzed in triplicate. At the end of the treatment, cell viability was examined with methyl thiazol tetrazolium (MTT) method according to the manufacturer's instructions. Briefly, $20 \mu \mathrm{L}$ reagent was added to each well and incubated at $37^{\circ} \mathrm{C}$ for another 4 hours. The reaction medium was removed and $150 \mu \mathrm{L}$ DMSO was added for dissolving the produced formazan crystals. Cell density was detected at $490 \mathrm{~nm}$ with a BioTek multifunctional microplate reader.

\section{RNA extraction and real-time PCR (RT-PCR)}

Total RNA was extracted from tissues or cells by using TRIzol solution (Life Technologies, Carlsbad, CA, USA). Reverse transcription was performed in a $10 \mu \mathrm{L}$ reaction system with $1 \mu \mathrm{g}$ RNA from samples to synthesize first-strand cDNA (Takara Bio Inc., Otsu, Shiga, Japan). The cDNA products were appropriately diluted for qPCR using KAPA qPCR Master Mix (Kapa Biosystems, Wilmington, MA, USA) and the primers used for human genes are listed in Table 1.

\section{Enzyme-linked immunosorbent assay (ELISA)}

Levels of dopamine in sera from 55 HCC patients and 18 healthy volunteers were detected with the Dopamine ELISA kit (R\&D System, Shanghai, People's Republic of China). The procedure was performed according to the instruction. A protocol for collecting blood samples from patients and volunteers was approved by the Board of Ethics Committee of the 10th People's Hospital. All of the written content were received before the test.

\section{Western blots}

Following treatment with or without THIO for 48 hours, the cells were collected using the lysis buffer $(25 \mathrm{mmol} / \mathrm{L}$ Tris [pH 6.8], 1\% sodium dodecyl sulfate, $5 \mathrm{mmol} / \mathrm{L}$ ethylene diamine tetraacetie acid, protease inhibitor cocktail [Sigma-Aldrich]). The protein concentrations of samples were determined by using the bicinchoninic acid kit, and sodium dodecyl sulfate-polyacrylamide gel electrophoresis was carried out for all the samples using the same amount of proteins. The protein membrane was blocked with 5\% nonfat milk in phosphate-buffered saline (PBS) containing $0.1 \%$ Tween and probed with the primary antibodies. The following primary antibodies were used in this study: CD133 (Miltenyi, Teterow, Germany), Caspase-3, EpCAM, and OCT4 antibodies (Cell Signaling Company, Danvers, MA, USA). IRDye-labeled secondary antibodies (Li-COR Biosciences, Lincoln, NE, USA) were used for detection. GAPDH was used as internal 
Table I Primer sequences for RT-PCR

\begin{tabular}{|c|c|c|c|}
\hline Gene name & Forward primer & Reverse primer & Size (bp) \\
\hline OCT4 & AAGGATGTGGTCCGAGTGTG & GAAGTGAGGGCTCCCATAGC & 180 \\
\hline SOX2 & ТТАССТСТТССТСССАСТССА & GGTAGTGCTGGGACATGTGAA & 132 \\
\hline NANOG & CTGTGATTTGTGGGCCTGAA & TGTTTGCCTTTGGGACTGGT & $|5|$ \\
\hline KLF4 & CTGCGGCAAAACCTACACAA & GGTCGCATTTTTGGCACTG & 182 \\
\hline Myc & TTCGGGTAGTGGAAAACCAG & CAGCAGCTCGAATTTCTTCC & 203 \\
\hline$D R I$ & GACCACCACAGGTAATGGAAAG & AAGAAAGGTAGCCAACAGCACA & $14 \mid$ \\
\hline DR5 & CTGGGCTAACTCCTCACTCAAC & ATTGCTGATGTTCACCGTCTC & 130 \\
\hline SNAI3 & ACTGCGACAAGGAGTACACC & GAGTGCGTTTGCAGATGGG & 220 \\
\hline Twist l & GGAGTCCGCAGTCTTACGAG & TCTGGAGGACCTGGTAGAGG & 201 \\
\hline E-cadherin & TGCCCAGAAAATGAAAAAGG & GTGTATGTGGCAATGCGTTC & 200 \\
\hline N-cadherin & ACAGCAACGACGGGTTAGTC & TCTGGGTCCTGAGCAGTGAA & 262 \\
\hline Slug & TGTGACAAGGAATATGTGAGCC & TGAGCCCTCAGATTTGACCTG & 203 \\
\hline GAPDH & AAGGTGAAGGTCGGAGTCAA & AATGAAGGGGTCATTGATGG & 108 \\
\hline Claudin I & ССTCCTGGGAGTGATAGCAAT & GGCAACTAAAATAGCCAGACCT & 145 \\
\hline FOXC2 & ССTCCTGGTATCTCAACCACA & GAGGGTCGAGTTCTCAATCCC & 134 \\
\hline MMP3 & CGGTTCCGCCTGTCTCAAG & CGCCAAAAGTGCCTGTCTT & 206 \\
\hline MMP9 & GGGACGCAGACATCGTCATC & TCGTCATCGTCGAAATGGGC & 139 \\
\hline
\end{tabular}

Abbreviation: RT-PCR, real-time polymerase chain reaction.

control. Density of each protein band was determined with Odyssey infrared imaging system (Li-COR).

\section{Cell cycle and apoptosis assays}

The LM3, Huh7, and SNU449 cells were seeded at a density of $3 \times 10^{5}$ cells/well in six-well plates and incubated overnight. The cells were treated with or without THIO for another 24 hours and harvested by trypsinization. For analysis of cell cycle, the cells were centrifuged at $1,000 \times g$ for 5 minutes and resuspended in PBS containing $5 \mathrm{mM}$ of NP-40 and RNase A $(1 \mathrm{mg} / \mathrm{mL})$. After incubation for 1 hour at $37^{\circ} \mathrm{C}$, the cells were labeled with propidium iodide (PI) for 15 minutes, and then were analyzed with FACSCanto II flow cytometer (BD Biosciences, Franklin lakes, NJ, USA). For analysis of apoptotic cells, the pellets were mixed in $100 \mu \mathrm{L}$ binding buffer containing Annexin-V/PI double staining reagents, and incubated at room temperature for 15 minutes in the dark. The stained cells were analyzed by flow cytometry and the percentage of apoptotic cells was measured using Diva software (Verity Software House, Topsham, ME, USA).

\section{Cell migration assay}

SNU449, Huh7, and LM3 cells were pretreated with or without THIO $(10 \mu \mathrm{M})$ for 72 hours in six-well plates, harvested, and resuspended in serum free DMEM with $0.1 \%$ bovine serum albumin. About $200 \mu \mathrm{L}$ of the cell suspension was added onto the insert filter at a density of $4 \times 10^{4}$ cells/ insert and then placed into the lower well containing $500 \mu \mathrm{L}$ of $10 \%$ FBS/DMEM. Transwell plates were incubated at $37^{\circ} \mathrm{C}$ with $5 \% \mathrm{CO}_{2}$ for 24 hours. Unmoved cells on the filters were removed using cotton swabs, and cells under the filters were fixed with dehydrated alcohol and stained with crystal violet. The number of migrating cells was counted under a microscope.

\section{Sphere formation}

SNU449, LM3, or Huh7 cells were suspended in serum-free stem cell medium containing DMEM/F12 (Gibco) supplemented with B27 supplement (Life Technologies), insulin (4 $\mu \mathrm{g} / \mathrm{mL}$; Sigma-Aldrich), EGF (20 ng/mL; PeproTech, Rocky Hill, NJ, USA), and bFGF (10 ng/mL; Life Technologies). Cells $(5,000,500$, and 100 cells per well) were seeded into ultralow-attachment six-well plates (Corning Life Sciences, Lowell, MA, USA) with or without THIO $(10 \mu \mathrm{M})$ treatment. After 7-10 days, the number of tumor spheres was photographed and counted under light microscopy (Leica Microsystems, Wetzlar, Germany).

\section{Xenograft tumor formation}

Five-week-old male BALB/c nude mice were purchased from B\&K Universal Company (Shanghai, People's Republic of China) and housed in the animal facility of the 10th People's Hospital under specific pathogen-free (SPF) conditions. The animal rooms were maintained on a 12-hours light/dark cycle, with standard conditions of light, food, and temperature. All the animal related procedures were approved by the Animal Care and Use Committee of The Tenth People's Hospital of Shanghai (document number 2011-RES1). The environment was maintained at a relative humidity of $30 \%-70 \%$. About $0.1 \mathrm{~mL}$ of LM3 cells $\left(1.0 \times 10^{6}\right)$ grown with DMEM or THIO for 3 days in serum-free culture medium was injected subcutaneously into the left or right upper flank region of nude mice respectively. Volumes of visible tumors were estimated at every 3rd day (length $\times$ width $\times$ width). When a subcutaneous tumor reached 
approximately $1.0 \mathrm{~cm}$ in diameter, the mice were sacrificed using $\mathrm{CO}_{2}$, and the tumors were isolated and measured.

\section{Results}

\section{Negative relationship between DR and dopamine in $\mathrm{HCC}$}

To understand the roles of DRs in the progression of HCC, we first detected expression of DRs in 32 pairs of human HCC specimens and nontumor adjacent tissues by RT-PCR with five pairs of primers for DRs, according to a previous report. ${ }^{17}$ The results exhibited that there were different levels of DRs in HCC. DR1 was upregulated in 12/32 pairs of tumor samples compared with nontumor adjacent tissues (Figure 1A) and DR5 was markedly upregulated in 20/32 tumor tissues (Figure 1B). But other types of DRs including DR2, DR3, and DR4 were too low to be detected.

In addition to levels of DR expression, we detected dopamine levels in the blood samples from HCC patients and normal healthy volunteers by ELISA. Levels of dopamine in the HCC patients were significantly lower when compared with blood samples from normal individuals (Figure 1C). However, dopamine increased cell proliferation
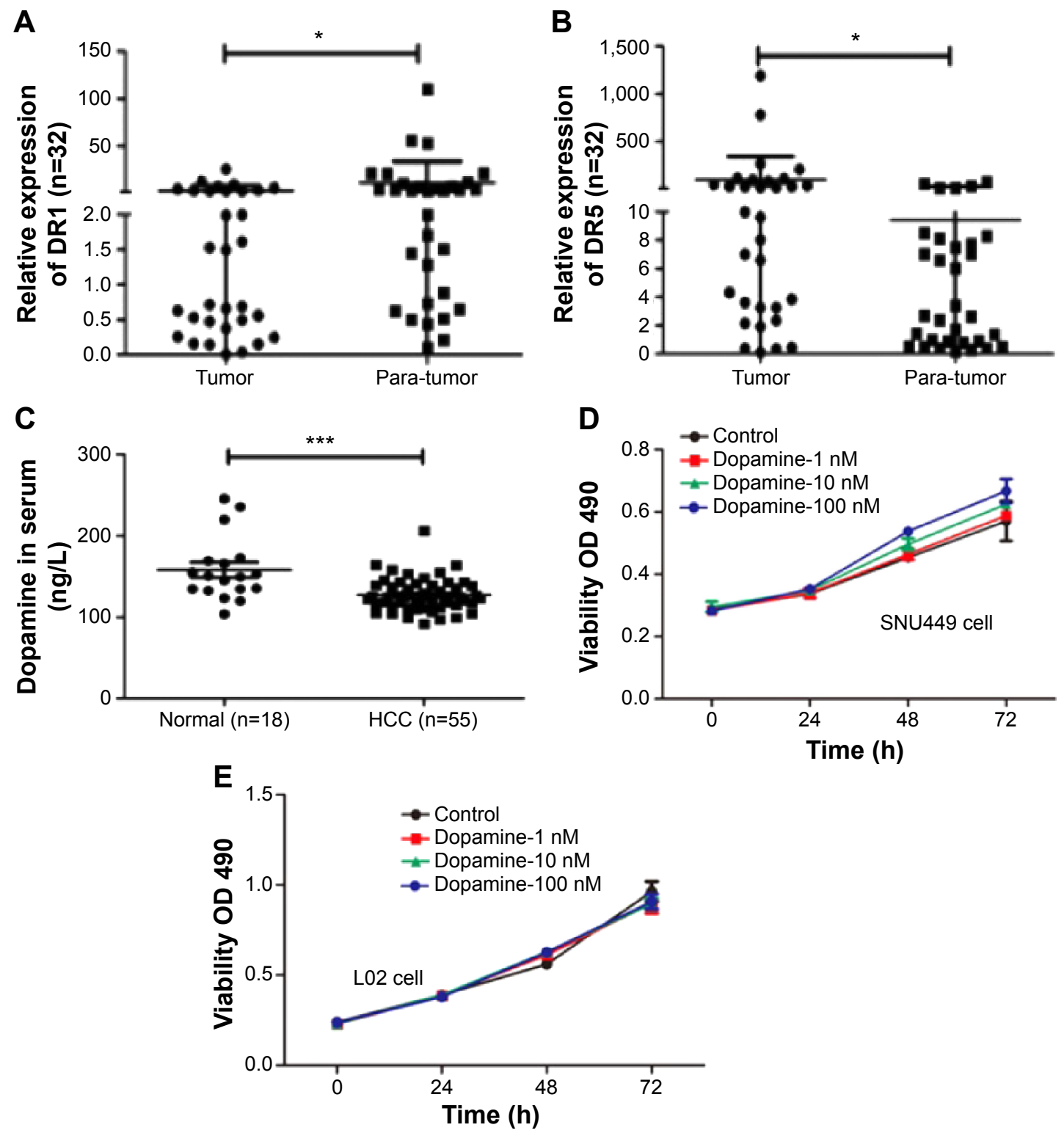

Figure I Negative relationship between dopamine receptor and dopamine in HCC.

Notes: (A) Levels of DRI was lower in HCC samples than those in tumor adjacent tissues. Thirty-two pairs of samples was detected in $\mathrm{qPCR}$ analysis. $* P<0.05$. (B) Levels of DR5 were higher in HCC samples than those in tumor adjacent tissues in qPCR analysis. Thirty-two pairs of samples was detected in qPCR analysis. *P $<0.05$. (C) Levels of dopamine in sera were detected by ELISA assay. ${ }^{* * * P}<0.005$. (D, E) Dopamine increased proliferation of SNU449 and L02 cells in the MTT assay.

Abbreviations: DR, dopamine receptor; ELISA, enzyme-linked immunosorbent assay; HCC, hepatocellular carcinoma; qPCR, quantitative polymerase chain reaction; MTT, methyl thiazol tetrazolium; OD, optical density. 
of SNU449 cells in a dose-dependent fashion, but had no effect on the growth of normal hepatic cell L02 in MTT assay (Figure 1D and E).

\section{DR antagonist THIO inhibits proliferation of hepatoma cells in vivo and in vitro}

THIO has been reported to inhibit tumor proliferation in several types of cancers, such as lung cancer, leukemia, breast cancer, colon cancer, etc. ${ }^{6-11}$ However, the role of THIO in liver cancer and other malignancies has not been reported. When we treated hepatoma cell lines SNU449, LM3, and Huh7 with THIO and compared them with normal hepatic cell L02, we found that THIO inhibited HCC cell growth in a dose-dependent fashion, but had slight inhibitiory role in $\mathrm{L} 02$ cell (Figure 2A). Optimal concentration for treatment of HCC cells in vitro was $10 \mu \mathrm{M}$. A concentration of $100 \mu \mathrm{M}$ caused cell death in 24 hours, but $5 \mu \mathrm{M}$ of THIO had a slight inhibitory effect (Figure 2A). To investigate the growth inhibitory mechanism of THIO, we observed the effect of the drug on cell cycle and apoptosis. By flow cytometry, we found that more of $\mathrm{HCC}$ cells were arrested in $\mathrm{G} 0 / \mathrm{G} 1$ phase after treatment with THIO for 24 hours (Figure 2B), suggesting that THIO inhibited cell proliferation via blocking the cell cycle. Interestingly, THIO appeared not to increase cell apoptosis (Figure 2B). To confirm this result, we further detected the cleaved Caspase- 3 after THIO treatment. The result showed no difference in expression levels of Procaspase-3 and cleaved Caspase- 3 between the THIO treatment group $(10 \mu \mathrm{M})$ and the DMSO group, in both LM3 and Huh-7 cells (Figure 2C).

To test if treatment with THIO would disrupt tumorigenicity of HCC cells, THIO- or DMSO-pretreated LM3 cells were subcutaneously injected into the left or right back of nude mice. The result showed that THIO-treated cells had smaller volume and weight of tumors than did DMSO-treated cells (Figure 2D-F), suggesting that THIO treatment could attenuate in vivo formation of xenograft tumors.

\section{THIO inhibits sphere formation and stemness genes expression in $\mathrm{HCC}$}

THIO has been reported to selectively induce differentiation of breast cancer stem cells. ${ }^{16}$ Thus, we investigated if blockage of DRs by THIO could inhibit the stemness property of HCC. Three HCC cell lines LM3, SNU449, and Huh7 were cultured in serum-free medium with or without THIO in an ultralow attachment plate. The number of spheres in each well was counted after random capture in four different locations. The result showed that DMSO-treated cells formed larger and more visible spheres, but THIO-treated cells had smaller or no spheres (Figure 3A). The results indicate that THIO could suppress the stem-like capacity of HCC cells.

Expression of stemness genes was further investigated. Western blotting showed that THIO decreased levels of
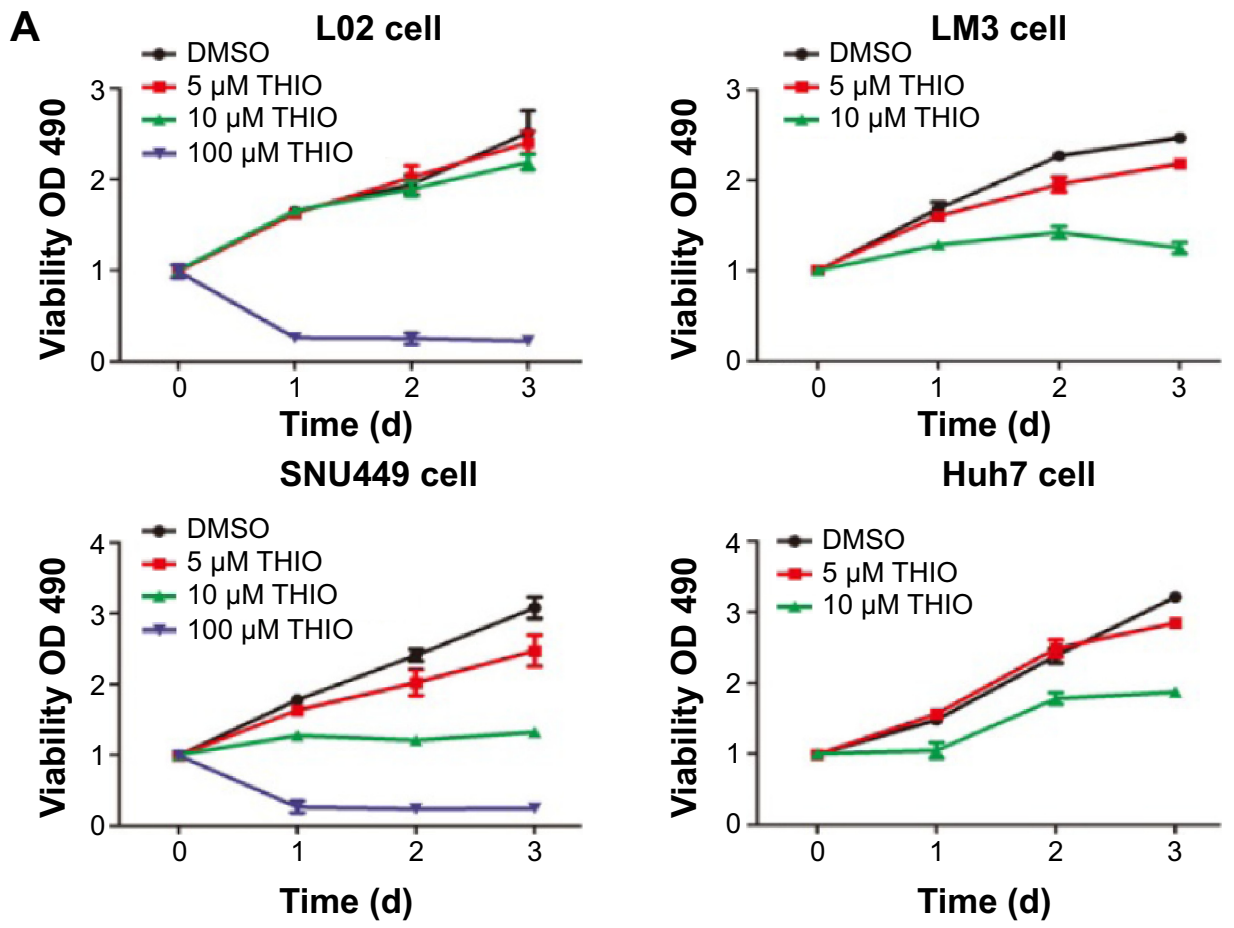

Figure 2 (Continued) 
B

\begin{tabular}{|c|c|c|c|c|c|}
\hline \multirow{2}{*}{$\begin{array}{l}\text { Cell } \\
\text { lines }\end{array}$} & \multirow{2}{*}{$\begin{array}{l}\text { Treatment } \\
\text { ( } \mu \mathrm{M} \text { thioridazine) }\end{array}$} & \multicolumn{3}{|c|}{ Cell cycle phase } & \multirow{2}{*}{$\begin{array}{l}\text { Apoptosis } \\
\text { Q4+Q2 (\%) }\end{array}$} \\
\hline & & G0/G1 (\%) & G2/M (\%) & S (\%) & \\
\hline \multirow{2}{*}{ SNU449 } & Control & 42.23 & 22.69 & 35.09 & 2.8 \\
\hline & THIO (10) & 56.35 & 21.13 & 22.52 & 2.8 \\
\hline \multirow{2}{*}{ LM3 } & Control & 44.81 & 25.48 & 29.71 & 3.8 \\
\hline & THIO (10) & 52.54 & 22.83 & 24.63 & 3.5 \\
\hline \multirow{2}{*}{ HUh7 } & Control & 73.62 & 6.59 & 19.78 & 0.4 \\
\hline & THIO (10) & 80.29 & 3.35 & 16.36 & 0.3 \\
\hline
\end{tabular}
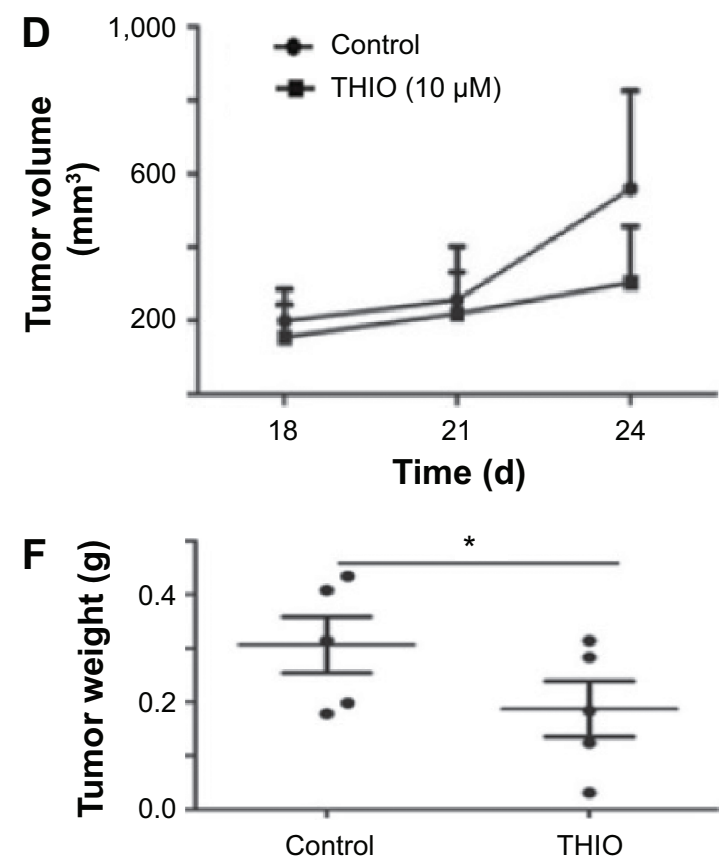

C

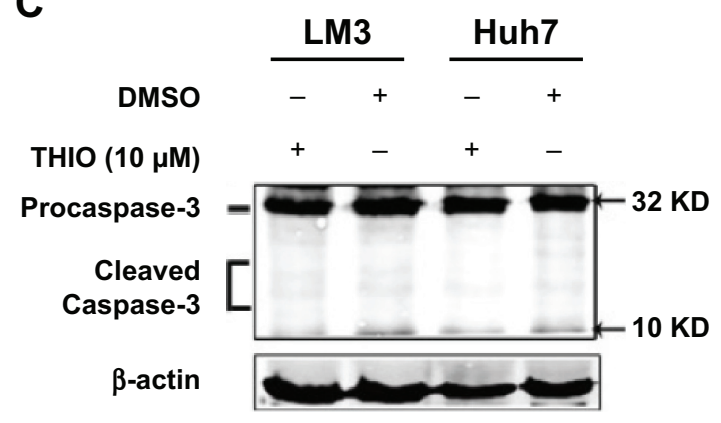

E

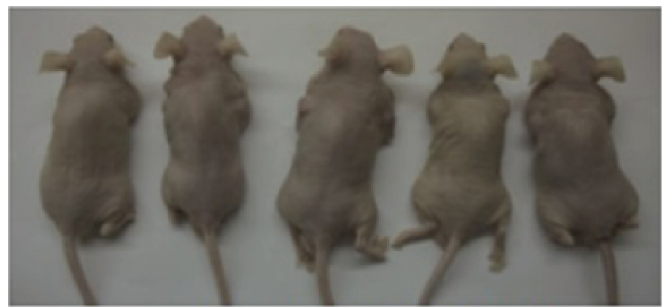

Control: right side; THIO: left side

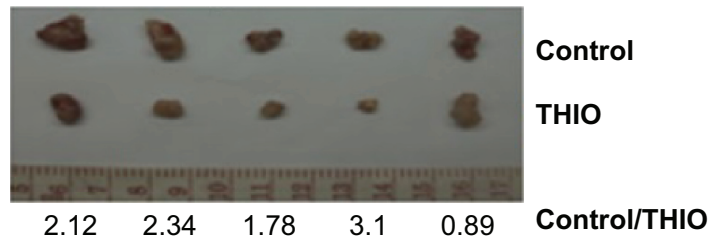

Figure 2 Thioridazine (THIO) inhibits proliferation of hepatoma cells in vivo and in vitro.

Notes: (A) Inhibitory effects of THIO on proliferation of HCC cell lines (SNU449, LM3, Huh7) and normal hepatic cell L02. (B) Effects of THIO on cell cycle progression and apoptosis of these HCC cell lines. (C) Western blot analysis of cleaved Caspase-3 expression in Huh7 and LM3 cells after treatments with DMSO or THIO. (D) Volumes of tumors were detected in mice spontaneously injected with LM3 cells pretreated with DMSO or $10 \mu M$ THIO. (E) Visible tumors were shown from mice at the 24 th day, the number below showed the ratio of tumor weight of Control over THIO group. (F) Weights of tumors from mice were measured at the 24 th day after injection. $* P<0.05$.

Abbreviations: HCC, hepatocellular carcinoma; DMSO, dimethyl sulfoxide; THIO, thioridazine; OD, optical density.

CD133, EpCAM, and OCT4 proteins in Huh7 cells compared with normal hepatocyte-like cells L02 (Figure 3B). It may be due to low or undetectable expression of DRs on L02 cells (data not shown). RT-PCR also revealed decreases of other stemness genes including SOX2, KLF4, MYC, and OCT4 in SNU449 and LM3 cells after THIO treatment (Figure 3C). Therefore, these results suggest that THIO inhibits cell proliferation possibly through suppressing the activities of the stem genes.

\section{THIO inhibit tumor cell migration through epithelial-mesenchymal transition (EMT)}

Cell capacity for motility plays a significant role during tumor progression and tumor metastasis. To determine if THIO treatment would affect HCC motility, we performed transwell assay for cells in the presence or absence of THIO. The results showed that transwell assay carried out on HCC cell lines (SNU449, LM3, Huh7) pretreated with THIO $(10 \mu \mathrm{M})$ for 72 hours showed reduced motility when compared with control cells (Figure 4A and B). With RT-PCR, expression of EMT- or motility-related genes, such as Claudin1, E-cadherin, N-cadherin, Twist1, Snail3, Slug, FOXC2, MMP3, MMP9, was studied, and THIO was found to decrease the expression of these genes in the three kinds of cell lines to different extents (Figure 4C-E). Therefore, THIO-mediated inhibition of cell movement may be dependent on reduction of migration-related gene expression.

\section{Discussion}

Dopamine, an important neurotransmitter, plays an important role in regulating gene expression, inducing cell 

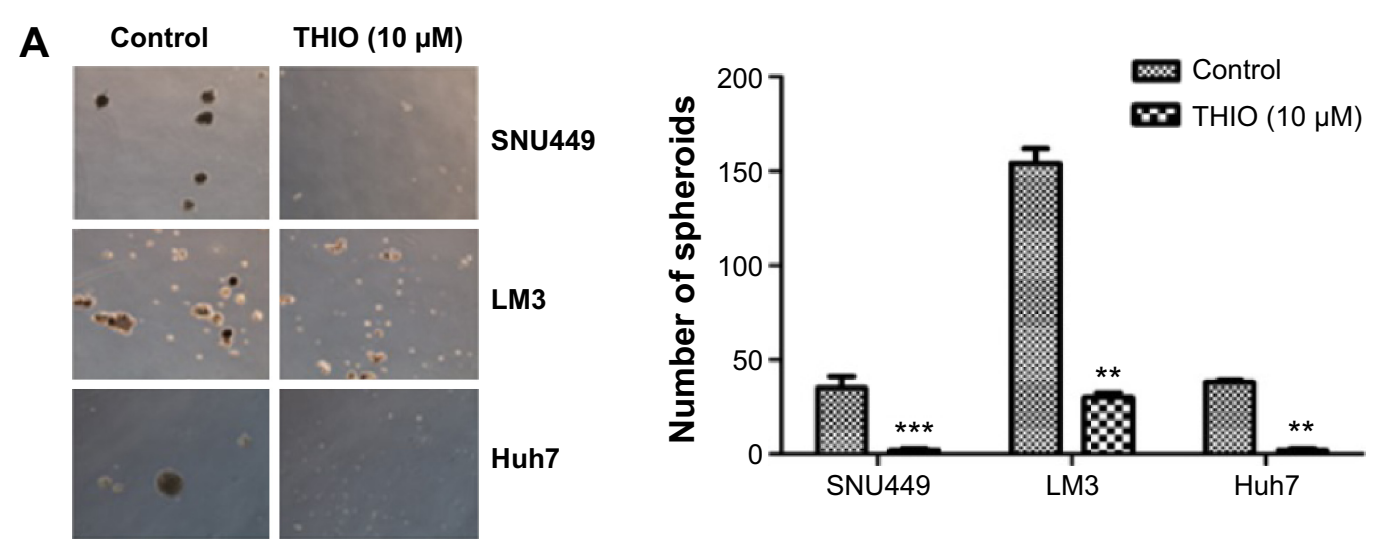

B

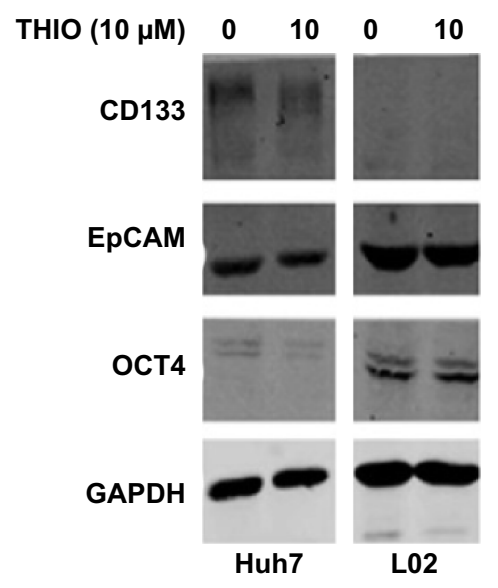

C

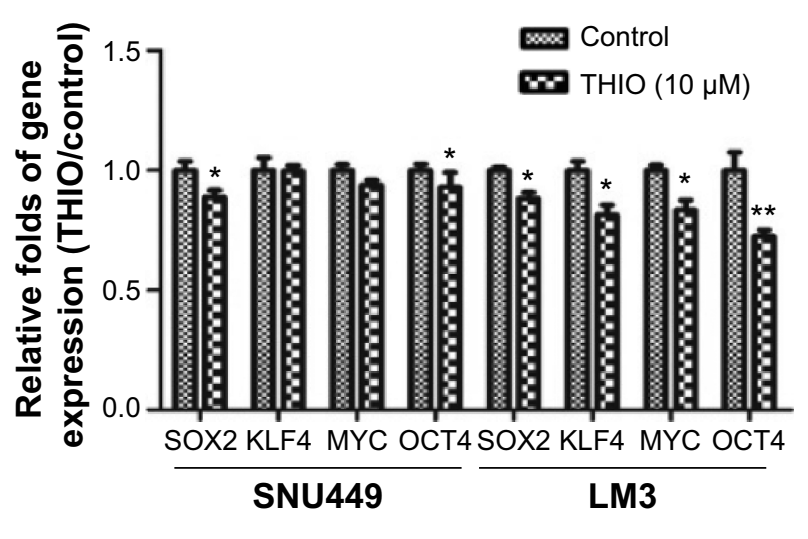

Figure 3 THIO inhibits sphere formation and stemness genes expression.

Notes: (A) Morphology (left) and quantification (right) of sphere formation in HCC cell lines after treatments with DMSO as Control or THIO (I0 $\mu$ M). (B) Western blot analysis of CDI33, EpCAM, and OCT4 expression in Huh7 and L02 cells after treatments with DMSO or THIO. (C) RT-PCR analysis for the stem cell markers in SNU449 and LM3 cells after treatments with DMSO or THIO. *P<0.05; **P<0.0I; ***P<0.005.

Abbreviations: HCC, hepatocellular carcinoma; RT-PCR, real-time polymerase chain reaction; DMSO, dimethyl sulfoxide; THIO, thioridazine.

proliferation and migration through activating its specific DRs. ${ }^{18}$ DRs are classified as D1 and D2 types. The D1 class includes the DR1 and DR5 subtypes, which are associated with activation of PKA signaling through increase of intracellular cAMP. ${ }^{2}$ The D2 class contains DR2, DR3, and DR4 subtypes, which are responsible for inhibition of intracellular cAMP. ${ }^{19}$ Dopamine can stabilize blood vessels through activation of pericyte cAMP-PKA signaling pathway by DR $1 .{ }^{20}$ Stressed mice with ovarian carcinoma have low levels of dopamine, and administered dopamine reduces the stimulatory effects of chronic stress on growth of ovarian cancer via inhibiting tumor angiogenesis and promoting tumor cell apoptosis through DR2. ${ }^{21}$ Our results show that levels of dopamine in the sera of HCC patients are lower than in the sera of normal healthy individuals. But treatment of HCC cells with dopamine increases cell proliferation, suggesting that dopamine is an important factor for tumor growth.
DRs are also expressed in different types of cancer to some extent. Human melanoma has no DR $1 .{ }^{22}$ Craniospinal and extracranial metastasis of a malignant prolactinoma have no DR2 protein, ${ }^{23}$ whereas high expression of DR2 was detected in a large series of neuroendocrine tumors. ${ }^{24}$ Further study showed that DR4 is repressed in central nervous system tumors of childhood, which eventually leads to inhibition of a DR4-mediated stimulation of the ERK1/2 kinase pathway. ${ }^{25}$ The DR D2 is involved in regulating Wnt and AKT signaling. ${ }^{26}$ However, the DR2 short isoform was considered as an inducer of apoptosis in lactotropes. ${ }^{27}$ Downregulation of DRs and increase of neuronal apoptosis are the consequences of the exposure of prenatal neurons to ethanol and pentylenetetrazol. ${ }^{28}$ Our studies reveal that relatively low DR1 and relatively high DR5 levels are found in HCC compared with the adjacent nontumor tissues. Others (DR2, DR3, and DR4) are too low to be detected. In HCC cell lines, LM3, SNU449, and Huh7 cells showed expression of 
A

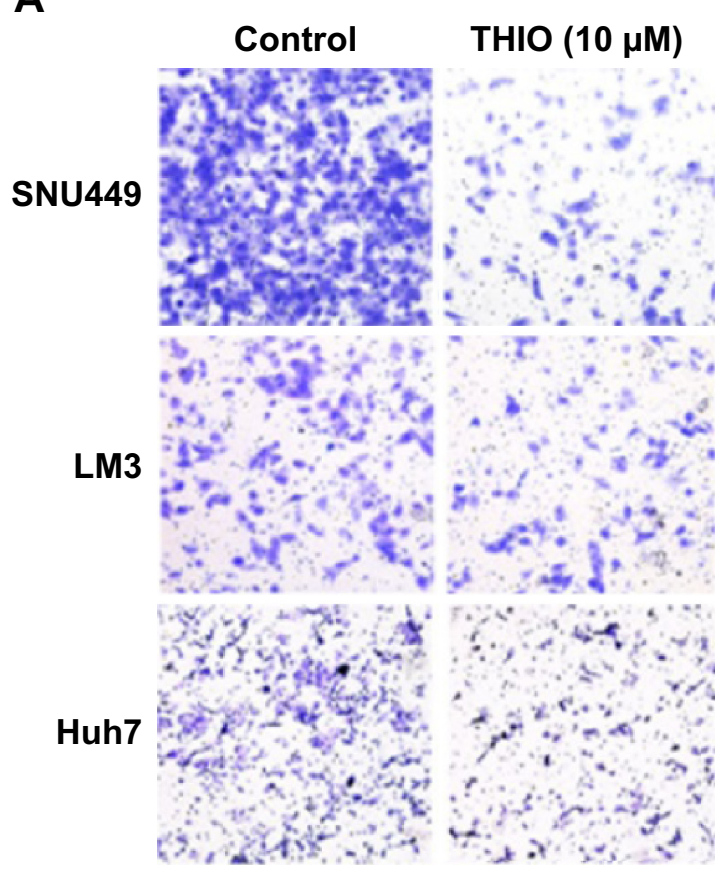

D

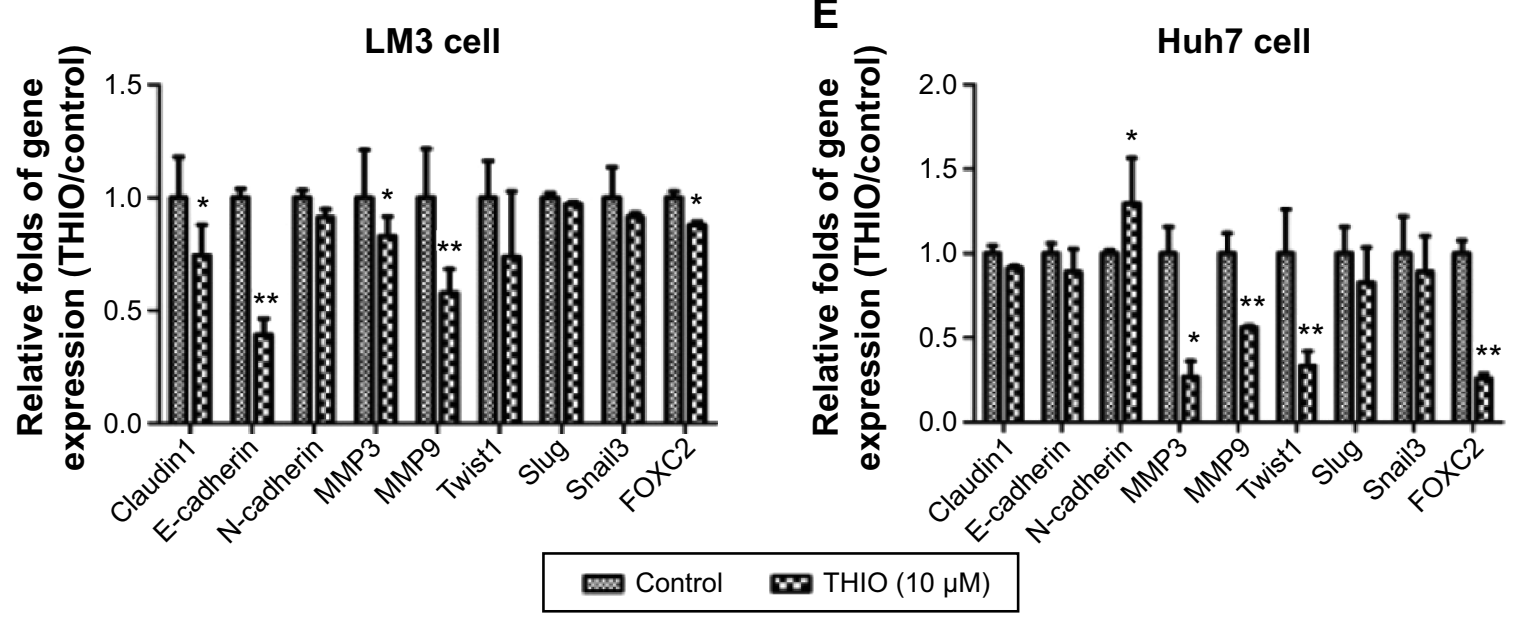

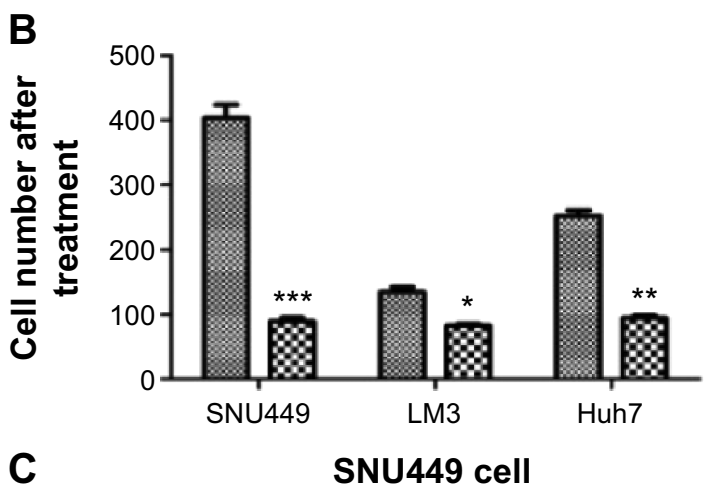

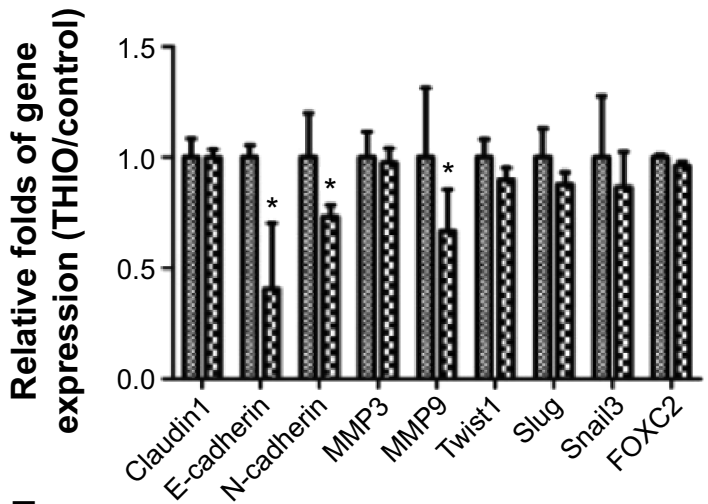

E

Figure 4 THIO inhibits tumor cell migration and expression of EMT-related genes.

Notes: (A) Transwell analysis for cells (SNU449, LM3, and Huh7) after treatments with THIO. (B) Quantification of migrated cells after transwell assay. $* P<0.05$, $* * P<0.01$, ***P $<0.005$. (C-E) RT-PCR analysis of the EMT-related genes in SNU449 (C), LM3 (D), and Huh7 (E) cells after treatment with THIO for 24 hours.

Abbreviations: RT-PCR, real-time polymerase chain reaction; THIO, thioridazine; EMT, epithelial-mesenchymal transition.

DR1- and DR2-like DRs. Blockage of DR by THIO exhibits inhibition of proliferation of $\mathrm{HCC}$ cells by inducing G0/G1 cell cycle arrest, whereas THIO did not show any apoptotic effects on the hepatoma cell lines just as other cancer cells do. Moreover, THIO also suppresses cell migration and sphere formation in vitro. Therefore, application of THIO may serve as an inhibitor for tumor metastasis.

The screening of many drugs for cancer has identified that antipsychotic drugs strongly inhibit cancer. Patients receiving DR antagonist medication were reported to have a decreased occurrence of colon, rectum, and prostate cancers. ${ }^{29}$
Dopaminergic-deficient Parkinson patients have lower cancer incidence rates. This suggests that DRs may be important in tumorigenesis. These findings are consistent with the lower levels of DRs seen in normal stem cell and higher levels seen in cancer stem cells. ${ }^{17}$ Previous studies have showed that THIO inhibited the PI3K/AKT pathway in cancer stem cells, ${ }^{7-11}$ which is consistent with our results that THIO decreases expression of stem genes and motility-related genes. Inhibition of DRs could therefore be a strategy for treatment of cancer.

The EMT is often activated during cancer invasion and metastasis and endows cells with migratory and invasive 
properties. ${ }^{30,31}$ EMT-related genes include Claudin1, Twist, Slug, Snail, E-cadherin, and N-cadherin. In addition, extracellular proteolysis also mediates tissue homeostasis. The matrix metalloproteinases (MMPs) represent the most prominent family of proteinases associated with tumorigenesis. ${ }^{30,32}$ THIO treatment in three HCC cell lines has displayed different reduction of the levels of these genes (Figure $4 \mathrm{C}-\mathrm{E}$ ), indicating that applying dopamine antagonist for HCC may help to reduce the risk of tumor metastasis. THIO has been shown to selectively target cancer stem cells in leukemia and breast cancer and induce cancer stem cells to differentiate. ${ }^{17}$ In this study, we determined that THIO can decrease mRNA levels of stem genes (OCT4, KLF4, SOX2, and $\mathrm{Myc})$. Therefore, further study will be focused on whether dopamine antagonists could help to increase efficiency of chemotherapy and reduce metastasis in vivo.

\section{Acknowledgments}

This work was supported by grants from the National Natural Science Foundation of China (81272427, 31471339 to ZL); National Key Basic Research Program of China (2013CB910500 to ZL); and a start-up grant from the 10th People's Hospital.

\section{Disclosure}

The authors report no conflicts of interest in this work.

\section{References}

1. Ferlay J, Shin HR, Bray F, et al. Estimates of worldwide burden of cancer in 2008: GLOBOCAN 2008. Int J Cancer. 2010;127:2893-2917.

2. Poon D, Anderson BO, Chen LT, et al. Management of hepatocellular carcinoma in Asia: consensus statement from the Asian Oncology Summit 2009. Lancet Oncol. 2009;10:1111-1118.

3. Yeh TC, Chiang PC, Li TK, et al. Genistein induces apoptosis in human hepatocellular carcinomas via interaction of endoplasmic reticulum stress and mitochondrial insult. Biochem Pharmacol. 2007;73:782-792.

4. Zhukovsky DS. Fever and sweats in the patient with advanced cancer. Hematol Oncol Clin North Am. 2002;16:579-588; viii.

5. Ly KL, Chidgey J, Addington-Hall J, Hotopf M. Depression in palliative care: a systematic review. Part 2. Treatment. Palliat Med. 2002;16: 279-284.

6. Mu J, Xu H, Yang Y, et al. Thioridazine, an antipsychotic drug, elicits potent antitumor effects in gastric cancer. Oncol Rep. 2014;31:2107-2114.

7. Kang S, Dong SM, Kim BR, et al. Thioridazine induces apoptosis by targeting the PI3K/Akt/mTOR pathway in cervical and endometrial cancer cells. Apoptosis. 2012;17:989-997.

8. Strobl JS, Kirkwood KL, Lantz TK, et al. Inhibition of human breast cancer cell proliferation in tissue culture by the neuroleptic agents pimozide and thioridazine. Cancer Res. 1990;50:5399-5405.

9. Gil-Ad I, Shtaif B, Levkovitz Y, et al. Characterization of phenothiazineinduced apoptosis in neuroblastoma and glioma cell lines: clinical relevance and possible application for brain-derived tumors. $J \mathrm{Mol}$ Neurosci. 2004;22:189-198.

10. Zhelev Z, Ohba H, Bakalova R, et al. Phenothiazines suppress proliferation and induce apoptosis in cultured leukemic cells without any influence on the viability of normal lymphocytes. Phenothiazines and leukemia. Cancer Chemother Pharmacol. 2004;53:267-275.
11. Rho SB, Kim BR, Kang S. A gene signature-based approach identifies thioridazine as an inhibitor of phosphatidylinositol-3'-kinase (PI3K)/AKT pathway in ovarian cancer cells. Gynecol Oncol. 2011; 120:121-127.

12. Byun HJ, Lee JH, Kim BR, et al. Anti-angiogenic effects of thioridazine involving the FAK-mTOR pathway. Microvasc Res. 2012; 84:227-234

13. Ke XY, Lin Ng VW, Gao SJ, et al. Co-delivery of thioridazine and doxorubicin using polymeric micelles for targeting both cancer cells and cancer stem cells. Biomaterials. 2014;35:1096-1108.

14. Tuynder M, Fiucci G, Prieur S, et al. Translationally controlled tumor protein is a target of tumor reversion. Proc Natl Acad Sci U S A. 2004; 101:15364-15369.

15. Amson R, Karp JE, Telerman A. Lessons from tumor reversion for cancer treatment. Curr Opin Oncol. 2013;25:59-65.

16. Sachlos E, Risueno RM, Laronde $\mathrm{S}$, et al. Identification of drugs including a dopamine receptor antagonist that selectively target cancer stem cells. Cell. 2012;149:1284-1297.

17. Neto LV, Machado Ede O, Luque RM et al. Expression analysis of dopamine receptor subtypes in normal human pituitaries, nonfunctioning pituitary adenomas and somatotropinomas, and the association between dopamine and somatostatin receptors with clinical response to octreotide-LAR in acromegaly. J Clin Endocrinol Metab. 2009;94(6):1931-1937.

18. Li L, Miyamoto M, Ebihara Y, et al. DRD2/DARPP-32 expression correlates with lymph node metastasis and tumor progression in patients with esophageal squamous cell carcinoma. World J Surg. 2006; 30:1672-1679; discussion 80-81.

19. Chakroborty D, Sarkar C, Basu B, et al. Catecholamines regulate tumor angiogenesis. Cancer Res. 2009;69:3727-3730.

20. Moreno-Smith M, Lee SJ, Lu C, et al. Biologic effects of dopamine on tumor vasculature in ovarian carcinoma. Neoplasia. 2013;15: $502-510$.

21. Moreno-Smith M, Lu C, Shahzad MM, et al. Dopamine blocks stress-mediated ovarian carcinoma growth. Clin Cancer Res. 2011;17: 3649-3659.

22. Boni R, Lichtensteiger W, Steinert HC, et al. D1 dopamine receptors are not expressed in human melanoma. Melanoma Res. 1997;7:117-119.

23. Winkelmann J, Pagotto U, Theodoropoulou M, et al. Retention of dopamine 2 receptor mRNA and absence of the protein in craniospinal and extracranial metastasis of a malignant prolactinoma: a case report. Eur J Endocrinol. 2002;146:81-88.

24. Grossrubatscher E, Veronese S, Ciaramella PD, et al. High expression of dopamine receptor subtype 2 in a large series of neuroendocrine tumors. Cancer Biol Ther. 2008;7:1970-1978.

25. Unland R, Kerl K, Schlosser S, et al. Epigenetic repression of the dopamine receptor D4 in pediatric tumors of the central nervous system. J Neurooncol. 2014;116:237-249.

26. Sutton LP, Rushlow WJ. The dopamine D2 receptor regulates Akt and GSK-3 via Dvl-3. Int J Neuropsychopharmacol. 2012;15:965-979.

27. Radl DB, Ferraris J, Boti V, et al. Dopamine-induced apoptosis of lactotropes is mediated by the short isoform of $\mathrm{D} 2$ receptor. PLoS One. 2011;6(3):e18097. doi:10.1371/journal.pone.0018097.

28. Naseer MI, Ullah I, Rasool M, et al. Downregulation of dopamine D1 receptors and increased neuronal apoptosis upon ethanol and PTZ exposure in prenatal rat cortical and hippocampal neurons. Neurol Sci. 2014;35(11):1681-1688.

29. Dalton SO, Mellemkjaer L, Thomassen L, et al. Risk for cancer in a cohort of patients hospitalized for schizophrenia in Denmark, 1969-1993. Schizophr Res. 2005;75:315-324.

30. Mani SA, Guo W, Liao MJ, et al. The epithelial-mesenchymal transition generates cells with properties of stem cells. Cell. 2008; 133:704-715.

31. Thiery JP, Acloque H, Huang RY, Nieto MA. Epithelial-mesenchymal transitions in development and disease. Cell. 2009;139:871-890.

32. Kessenbrock K, Plaks V, Werb Z. Matrix metalloproteinases: regulators of the tumor microenvironment. Cell. 2010;141:52-67. 


\section{Publish your work in this journal}

OncoTargets and Therapy is an international, peer-reviewed, open access journal focusing on the pathological basis of all cancers, potential targets for therapy and treatment protocols employed to improve the management of cancer patients. The journal also focuses on the impact of management programs and new therapeutic agents and protocols on

patient perspectives such as quality of life, adherence and satisfaction. The manuscript management system is completely online and includes a very quick and fair peer-review system, which is all easy to use. Visit http://www.dovepress.com/testimonials.php to read real quotes from published authors.

Submit your manuscript here: http://www.dovepress.com/oncotargets-and-therapy-journal 